\title{
MODEL PENYEBARAN PENYAKIT MENULAR DENGAN TRANSMISI VERTIKAL
}

\author{
Usman Pagalay \\ Jurusan Matematika, Fakultas Sains dan Teknologi \\ Universitas Islam Negeri (UIN) Maulana Malik Ibrahim Malang \\ e-mail: usmanpagalay@yahoo.co.id
}

\begin{abstract}
The dynamics of many epidemic models for infectious diseases that spread in a single host population demonstrate a threshold phenomenon. If the basic reproduction number $R_{0}$ is below unity, the disease free equilibrium $P$ is globally stable in the feasible region and the disease always dies out. If $R_{0}>1$, a unique endemic equilibrium $P$ is globally asymptotically stable in the interior of the feasible region and the disease will persist at the endemic equilibrium if it is initially present. In this paper this threshold phenomenon is established for two epidemic models of SEIR type using two recent approaches to the global-stability problem.
\end{abstract}

Key words: Epidemic models, endemic equilibrium, latent period global stability.

\section{Pendahuluan}

Penyakit Menular dianggap sebagai penyakit yang serius dan korbannya mencapai 2,5 Miliar orang diseluruh dunia, khususnya pada daerah tropis. Rata-rata kematian yang terinfeksi penyakit ini berkisar antara 40\%.. Meskipun hampir semua penyakit Menular ini terjadi pada daerah tropis. Beberapa penelitian menunjukkan bahwa penyakit Menular mungkin saja terjadi di daerah dingin.

Untuk mengontrol penyakit Menular secara efektif, kita akan memahami dinamika penyebaran penyakit dan mempertimbangkan semua hal yang terkait secara detail dan menggunakan model Matematika dengan bantuan program computer.

\section{Model Matematika}

Pada tulisan ini, kita menganggap model SEIR yang memiliki angka kelahiran yang bersifat ekponensial dan diasumsikan bahwa penyakit menyebar melalui 2 arah (transmisi) yaitu transmisi horizontal dan transmisi vertikal.

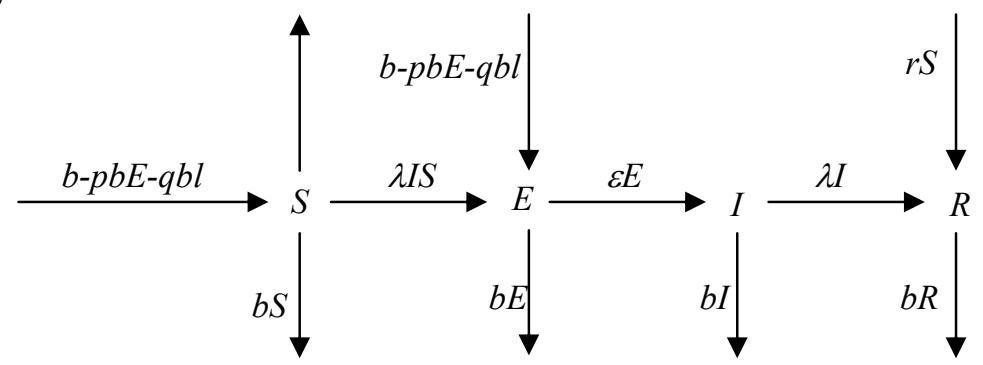

GAMBAR 1. Alur diagram antar kompartemen

Diasumsikan bahwa penyakitnya tidak fatal $(\alpha=0)$, tingkat kelahiran dan kematian sama dan dinotasikan $b$. oleh karena itu, total populasinya $(N)$ konstan, yaitu $N=S+E+I+R=1$, dan maka dari itu angka kelahiran Asli $A+b N=b$. Pada transmisi vertikal, kita asumsikan bahwa pecahan $p$ dan $q$ pada turunan dari bagian exposed dan infection, menghasilkan bagian $E$. konsekuensinya perubahan kelahiran pada kelas $E$ (exposed) diberikan oleh persamaan $p b E+q b I$ sehingga perubahan kelahiran pada bagian $S$ (suscepsible) diberikan oleh persamaan $b-p b E-q b I$ dengan 
$0 \leq p \leq 1$ dan $0 \leq q \leq 1$. Pada vaksinasi percacaran kita asumsikan bahwa semua individu yang rentang terhadap penyakit, diberi vaksinasi dengan nilai tetap $r$, dan vaksinasi tidak memiliki efek pada individu yang terinveksi. Jika $r=0$, maka tidak ada vaksinasi yang diperhitungkan. Transfer diagram akan diperlihatkan pada sistem persamaan differensial berikut:

$$
\begin{aligned}
& S^{\prime}=b-\lambda i S-p b E-q b i-b S-r S \\
& E^{\prime}=\lambda i S+p b E+q b i-(\varepsilon+b) E \\
& i^{\prime}=\varepsilon E-(\gamma+b) i \\
& R^{\prime}=\gamma i-b R+r S
\end{aligned}
$$

Dalam analisa dinamik, kompartemen removed tidak berpengaruh sehingga dapat ditulis:

$$
\begin{aligned}
& S^{\prime}=b-\lambda i S-p b E-q b i-b S-r S \\
& E^{\prime}=\lambda i S+p b E+q b i-(\varepsilon+b) E \\
& i^{\prime}=\varepsilon E-(\gamma+b) i
\end{aligned}
$$

Notasi domain adalah $\sum=\left\{(S, E, i) \in R^{3} .: S+E+i \leq 1\right\}$. Dinamika dari (1.2) ditentu-kan oleh teori Basic Reproduction Number yang dimodifikasi menjadi :

$$
R_{0}=\frac{\lambda \varepsilon}{(b+\varepsilon)(b+\gamma)-b p(b+\gamma)-b q \varepsilon} \frac{b}{b+r}
$$

jika $R_{0} \leq 1$, maka penyakit akan hilang dan keseimbangannya adalah $P_{0}=\{b /(b+r), 0,0\}$. Jika $R_{0}>1$ maka keseimbangan endemic $P^{*}=\left\{S^{*}, E^{*}, i^{*}\right\}$ dengan $S^{*}=1 / \mathrm{R}_{0}$.

Berikut adalah hasil yang dapat dibuktikan sebagai proposisi.

Pandang sistem persaman berikut

$$
\begin{aligned}
& S^{\prime}=A-d S-\lambda i S \\
& E^{\prime}=\lambda i S-(\varepsilon+d) E \\
& i^{\prime}=\varepsilon E-(\gamma+a+d) i
\end{aligned}
$$

Digunakan sebuah fungsi Lyapunov $L=\varepsilon E+(\varepsilon+b-p b) i$.

Bukti:

Matriks jacobian $\quad J 1=\frac{\partial J}{\partial x}$ untuk persamaan (1.2) dengan sebuah solusi umum $\{\mathrm{S}(\mathrm{t}), \mathrm{E}(\mathrm{t}), \mathrm{i}(\mathrm{t})\}$ adalah

$$
J 1=\left[\begin{array}{ccc}
-\lambda i-b-r & -p b & -\lambda S-q b \\
\lambda i & p b-\varepsilon-b & \lambda S \\
0 & \varepsilon & -\lambda-b
\end{array}\right]
$$

dan untuk nilai $\mathrm{J}^{2}$ adalah berdasarkan rumus untuk mencari $\mathrm{J}^{2}$ adalah

$$
J^{|2|}=\left[\begin{array}{ccc}
a_{11}+a_{22} & a_{23} & -a_{13} \\
a_{32} & a_{11}+a_{33} & a_{12} \\
-a_{31} & a_{21} & a_{22}+a_{33}
\end{array}\right]
$$

Maka dari persamaan (1.4) kita dapatkan untuk $\mathrm{J}^{2}$-nya adalah

$$
J^{|2|}=\left[\begin{array}{ccc}
-\lambda i-2 b-r+p b-\varepsilon & \lambda S-q b & \lambda S+q b \\
\varepsilon & -\lambda i-2 b-r-\lambda & -p b \\
0 & \lambda i & p b-\varepsilon-2 b-\lambda
\end{array}\right]
$$


Definisikan fungsi

dimana

$$
B=P_{f} P^{-1}+P \frac{\partial f^{|2|}}{\partial x} P^{-1}
$$

$$
P(S, E, i)=\left[\begin{array}{ccc}
a_{1} & 0 & 0 \\
0 & \frac{\left(1-\alpha_{2}\right) E}{i} & 0 \\
0 & \frac{a_{2} E}{i} & \frac{E}{i}
\end{array}\right]
$$

dan $1<a_{1}<1+\lambda c^{2} /(\lambda+b)$, c adalah konstan dan

$$
a_{2}= \begin{cases}0, & \text { jika } \varepsilon \geq p b \\ 1-\frac{\varepsilon}{p b}, & \text { jika } \varepsilon<p b\end{cases}
$$

sehingga dapat ditulis dalam bentuk $B=\left[\begin{array}{ll}B_{11} & B_{12} \\ B_{21} & B_{22}\end{array}\right]$ dengan

$$
\begin{aligned}
& B_{11}=-\lambda i-\varepsilon-2 b-r-p b \quad B_{12}=a_{1}\left[\frac{(\lambda S+q b) I}{E}, \frac{(\lambda S+q b) i}{E}\right], \\
& B_{21}=\frac{1}{a_{1}}\left[\begin{array}{c}
\left(1-a_{2}\right) \frac{\varepsilon E}{i} \\
a_{2} \frac{\varepsilon E}{i}
\end{array}\right], \text { dan } \\
& B_{22}=\left[\begin{array}{cc}
\frac{E^{\prime}}{E}-\frac{i^{\prime}}{i}-\lambda i-\gamma-2 b-r+a_{2} p b \\
\lambda i+\frac{a_{2}\left[\varepsilon-\left(1-a_{2}\right) p b\right]}{1-a_{2}} & \frac{E^{\prime}}{E}-\frac{i^{\prime}}{i}-\varepsilon-\gamma-2 b+\left(1-a_{2}\right) p b
\end{array}\right]
\end{aligned}
$$

yang dapat disederhanakan menjadi

$$
\left[\begin{array}{cc}
\frac{E^{\prime}}{E}-\frac{i^{\prime}}{i}-\lambda i-\gamma-2 b-r+a_{2} p b & -\left(1-a_{2}\right) p b \\
\lambda i & \frac{E^{\prime}}{E}-\frac{i^{\prime}}{i}-\varepsilon-\gamma-2 b+\left(1-a_{2}\right) p b
\end{array}\right]
$$

gunakan $a_{2} \leq-b / 2$ dari $\bar{q}_{2}=\operatorname{limsupsup}_{t \rightarrow \infty} \frac{1}{t} \int_{0}^{t} \mu(B(x(s, x 0))) d s$.

jika $\mathrm{r}=0$ kemudian $\mathrm{R}_{0}$ mengurangi parameter buka $\mathrm{R}_{0}(\mathrm{p}, \mathrm{q})$ untuk sebuah model SEIR dengan tanpa vaksinasi. jika $R_{0}=\frac{b}{b+r} R_{0}(p, q)$, maka hubungan ini sebenarnya menjelaskan bahwa vaksinasi rendah sehinggsa nilai dasar reproduksinya juga rendah. Jika $\mathrm{r}=\mathrm{p}=\mathrm{q}=0$, maka diberikan $R_{0}=\frac{\lambda \varepsilon}{(\varepsilon+b)(\gamma+b)}$. Didalam batasan tersebut berlaku $\quad \varepsilon \rightarrow \infty$, sehingga $\mathrm{R}_{0}$ memberikan dasar reproduksi bilangan untuk sebuah model SIR. 


\section{Hasil Numerik}

Diberikan Nilai $\mathrm{d}=0,8 ; \lambda=0,4 ; \mathrm{A}=0,16 ; \varepsilon=0,03 ; \gamma=\mathrm{a}=0$.

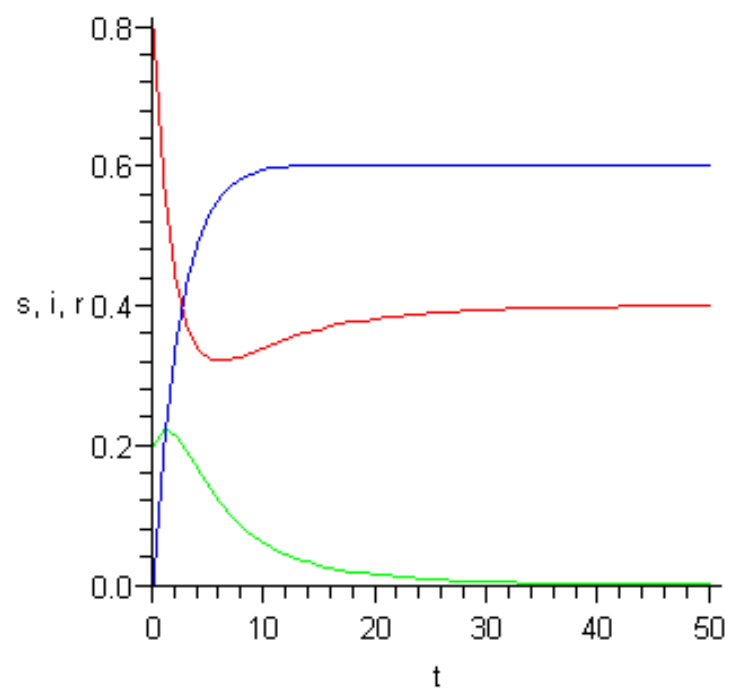

GAMBAR 2. Perilaku $S(\mathrm{t})$ (Merah) pada hari pertama terlihat turun dan kemudian cenderung tetap, dengan nilai awal 0.8; Perilaku E(t) (hijau) pada hari pertama terlihat naik dan kemudian cenderung tetap dengan nilai awal 0.2; Perilaku $I(\mathrm{t})$ (biru) pada hari pertama terlihat Naik dan kemudian cenderung tetap dengan nilai awal 0.1. Ketiga grafik tersebut menuju titik equilibrium.

\section{Kesimpulan}

Jika $\lambda$ dan $\varepsilon$ membesar maka $R_{0}$ juga membesar. Artinya dalam mengontrol penyakit menular, perlu diperhatikan nilai $\lambda$ dan $\varepsilon$.

\section{Daftar Pustaka}

Anderson, R.M. and May, R.M., (1992), Infectious Diseases of Humans Dynamics and Control, Oxford University Press, Oxford.

Anderson, R.M. and May, R.M., Population biology of infectious diseases I, 180(179) Nature pp.361-367.

Busenberg, S. and Cooke, K., (1993), Vertically Transmitted Diseases, Biomathematics, Vol.23, Springer-Verlag, Berlin.

Coppel, W.A., (1965), Stability and Asymptotic Behavior of Differential Equations, Health, Boston.

Esteva, L. and Vargas, C., (1990), A model for dengue disease with variable human population, J. Math. Biol., 3-8, Pp.220-240.

Hale, J.K., (1969), Ordinary Differential Equations, John Wiley \& Sons, New York.

Hethcote, H.W., and S.A., Levin Periodicity in epidemiological models in Applied Mathematical Ecology, L, Gross and S.A. Levineds Springer, New York, pp.193211.

Hethcote, H.W., Stech, H.W., and Van den Driessche, P., (1981), Periodicity and stability in epidemic models a survey in Differential Equations and Applications in Ecology Epidemics and Population Problems, K.L.Cook ed Academic Press, New York, pp.65-85.

Li, M.Y. and Muldowney, J.S., (1995), Global stability for the SEIR model in epidemiology, Math.Biosci., 125, pp.155-164. 
Li, M.Y., Graef, J.R., Wang, L.C., and Karsai, J., (1999), Global dynamics of a SEIR model with a varying total population size, Math. Biosci., 160, pp.191-213.

Li, M.Y., Smith, H.L., and Wang, L., Global stability of an SEIR model with vertical transmission, SIAM. J. Appl.Math., to appear.

\section{Lampiran}

Program Maple untuk melihat Matriks pelinearan dari sistem

$>$ restart:with(plots):with(linalg):

$>d S:=b-1 a m b d a * i * S-p * b * E-q * b * i-b * S-r * S$;

$d E:=l a m b d a * i * S+p * b * E+q * b * 1-($ epsilon $+b) * E$;

di: =epsilon*E- $($ lambda $+b) * i$;

$$
\begin{gathered}
d S:=b-\lambda i S-p b E-q b i-b S-r S \\
d E:=\lambda i S+p b E+q b l-(\varepsilon+b) E \\
d i:=\varepsilon E-(\lambda+b) i
\end{gathered}
$$

$>\mathbf{R}[0]:=($ lambda*epsilon $) /((b+e p s i l o n) *(b+l a m b d a)-$

$\left.b^{*} p *(b+l a m b d a)-b * q * e p s i l o n\right) * b /(b+r)$;

$$
R_{0}:=\frac{\lambda \varepsilon b}{((\varepsilon+b)(\lambda+b)-b p(\lambda+b)-b q \varepsilon)(b+r)}
$$

$>\mathrm{j} 1:=\mathrm{jacobian}([\mathrm{dS}, \mathrm{dE}, \mathrm{di}],[\mathrm{S}, \mathrm{E}, \mathrm{i}])$;

$$
j 1:=\left[\begin{array}{ccc}
-\lambda i-b-r & -p b & -\lambda S-q b \\
\lambda i & p b-\varepsilon-b & \lambda S \\
0 & \varepsilon & -\lambda-b
\end{array}\right]
$$

dengan menggunakan rumus untuk mencari $\mathrm{J} 2$, maka didapat nilai $\mathrm{j} 2$ sebagai berikut :

$>j 2:=\operatorname{matrix}(3,3,[[-1 a m b d a * i-b-r+p * b-e p s i l o n-b$, lambda* $\left.S+q * b,-\left(-1 a m b d a * S-q^{*} b\right)\right]$, [epsilon, - lambda*i-b-r+ $(-1 a m b d a-b,-p * b],\left[-0,1 a m b d a * i, p^{*} b-e p s i l o n-b+(-1 a m b d a-\right.$ b) ] ] ) ;

$$
j 2:=\left[\begin{array}{ccc}
-\lambda i-2 b-r+p b-\varepsilon & \lambda S-q b & \lambda S+q b \\
\varepsilon & -\lambda i-2 b-r-\lambda & -p b \\
0 & \lambda i & p b-\varepsilon-2 b-\lambda
\end{array}\right]
$$

$>\mathrm{P}(\mathrm{S}, \mathrm{E}, \mathrm{i})=\operatorname{Matrix}\left(3,3,\left[[\mathrm{a}[1], 0,0],\left[0,\left((1-\mathrm{a}[2]){ }^{*} \mathrm{E} / \mathrm{i}\right), 0\right]\right.\right.$, $\left.\left.\left[0, a[2]{ }^{*} E / i, E / i\right]\right]\right)$;

$$
P(S, E, i):=\left[\begin{array}{ccc}
a_{1} & 0 & 0 \\
0 & \frac{\left(1-\alpha_{2}\right) E}{i} & 0 \\
0 & \frac{a_{2} E}{i} & \frac{E}{i}
\end{array}\right]
$$

$>\mathrm{B}=\operatorname{Matrix}(2,[[\mathrm{~B}[11], \mathrm{B}[12]],[\mathrm{B}[21], \mathrm{B}[22]]])$;

$$
B=\left[\begin{array}{ll}
B_{11} & B_{12} \\
B_{21} & B_{22}
\end{array}\right]
$$

$>B[11]:=-$ lambda*i-epsilon $-2 * b-r-p * b$;

$>B[12]:=a[1] *[(1 a m b d a * S+q * b) * I / E,(l a m b d a * S+q * b) * i / E]$;

$>B[21]:=[1 / a[1]]^{*} \operatorname{matrix}([[(1 a[2]) * e p s i l o n * E / i]$,

[a[2]*epsilon*E/i]]);

Volume 1 No. 1 November 2009 
$B$ [22]: =Matrix $(2,2,[[e / E-I / i-l a m b d a * i-g a m m a-2 * b-r+a[2] * p b$, $-(1-a[2]) * p * b],[$ lambda* $i+a[2] *$ [epsilon $-(a-a[2]) * p * b] /$ $\left.\left.\left.(1-a[2]), e / E-I / i-e p s i l o n-g a m m a-2 * b+(1-a[2])^{*} p^{*} b\right]\right]\right)$;

$$
\begin{gathered}
B_{11}:=-\lambda i-\varepsilon-2 b-r-p b \\
B_{12}:=a_{1}\left[\frac{(\lambda S+q b) I}{E}, \frac{(\lambda S+q b) i}{E}\right], \\
B_{21}:=\left[\frac{1}{a_{1}}\right]\left[\begin{array}{c}
\left.\frac{\left(1-a_{2}\right) \varepsilon E}{i}\right] \\
\frac{a_{2} \varepsilon E}{i}
\end{array}\right] \\
B_{22}:=\left[\begin{array}{cc}
\frac{e}{E}-\frac{I}{i}-\lambda i-\gamma-2 b-r+a_{2} p b \\
\lambda i+\frac{a_{2}\left[\varepsilon-\left(a-a_{2}\right) p b\right]}{1-a_{2}} & \left.\frac{e}{E}-\frac{I}{i}-\varepsilon-\gamma-2 b+\left(1-a_{2}\right) p b\right]
\end{array}\right.
\end{gathered}
$$

